\title{
3 Research Square \\ Shared Genetic Loci For Body Fat Storage And Adipocyte Lipolysis In Humans
}

\section{Agné Kulyté}

Karolinska Institutet

\section{Veroniqa Lundbäck}

Karolinska Institutet

Peter Arner

Karolinska Institutet

\section{Rona J. Strawbridge}

University of Glasgow

Ingrid Dahlman ( $\nabla$ ingrid.dahlman@ki.se )

Karolinska Institutet

\section{Research Article}

Keywords: genetic variants, lipolysis, body mass index, waist-hip ratio, adipocytes, gene expression, genome-wide association studies

Posted Date: September 7th, 2021

DOl: https://doi.org/10.21203/rs.3.rs-810555/v1

License: (c) (i) This work is licensed under a Creative Commons Attribution 4.0 International License. Read Full License

Version of Record: A version of this preprint was published at Scientific Reports on March 7th, 2022. See the published version at https://doi.org/10.1038/s41598-022-07291-4. 


\section{Abstract}

Background. Total body fat and central fat distribution are heritable traits and well-established predictors of adverse metabolic outcomes. Lipolysis is the process responsible for the hydrolysis of triacylglycerols stored in adipocytes. To increase our understanding of the genetic regulation of body fat distribution and total body fat, we set out to determine if genetic variants associated with body mass index (BMI) or waisthip-ratio adjusted for BMI (WHRadjBMI) in genome-wide association studies (GWAS) mediate their effect by influencing adipocyte lipolysis.

Methods. We utilized data from the recent GWAS of spontaneous and isoprenaline-stimulated lipolysis in the unique GENetics of Adipocyte Lipolysis (GENiAL) cohort. GENiAL consists of 939 participants who have undergone abdominal subcutaneous adipose biopsy for the determination of spontaneous and isoprenaline-stimulated lipolysis in adipocytes.

Results. We report $11 \mathrm{BMI}$ and 15 WHRadjBMI loci with SNPs displaying nominal association with lipolysis and allele-dependent gene expression in adipose tissue according to in silico analysis.

Functional evaluation of candidate genes in these loci by small interfering RNAs (siRNA)-mediated knockdown in adipose-derived stem cells identified ZNF436 and NUP85 as intrinsic regulators of lipolysis consistent with the associations observed in the clinical cohorts. Furthermore, candidate genes in another BMI-locus (STX17) and two more WHRadjBMI loci (NID2, GGA3, GRB2) control lipolysis alone, or in conjunction with lipid storage, and may hereby be involved in genetic control of body fat.

Conclusions. The findings expand our understanding of how genetic variants mediate their impact on the complex traits of fat storage and distribution.

\section{Introduction}

Obesity, with abdominal fat deposition, is a risk factor for common metabolic diseases. Both overall obesity, as assessed by body mass index (BMI), and a central fat deposition, as assessed by waist-hip ratio adjusted for BMI (WHRadjBMI), display strong genetic predisposition 1,2. By genome-wide association studies (GWAS) numerous genetic risk loci for BMI and body fat distribution have been identified ${ }^{1,2}$. In most cases, the underlying susceptibility genes in these loci and regulatory pathways remain to be defined.

One mechanism in adipose tissue implicated in the control of fat mass is hydrolysis of triglycerides, i.e., lipolysis. High spontaneous and low catecholamine-stimulated lipolysis are associated with higher body weight in both cross-sectional and prospective cohorts ${ }^{3,4}$. We have recently reported the first GWAS of abdominal spontaneous and isoprenaline-stimulated adipose lipolysis in the unique GENiAL cohort ${ }^{5}$.

The present study aimed to determine whether genetic variants, identified by GWAS, associate with BMI 6 and central fat deposition (e.g. WHRadjBMI) ${ }^{7}$ overlap with those lipolysis-associated ${ }^{5}$. Overlapping risk alleles for these traits were examined for allele-dependent gene expression through in silico analysis. 
Genes with allele-dependent expression patterns in adipose tissue were functionally evaluated in human adipose derived mesenchymal stem cells (hASCs).

\section{Materials And Methods Participants}

The GENiAL cohort and GWAS of abdominal subcutaneous adipose tissue (SAT) lipolysis have been described previously ${ }^{5}$. WHRadjBMI was calculated in a sex-specific manner by inverse-normal transformation of the residuals of the linear regression model: WHR adjusted for age, age ${ }^{2}$ and $\mathrm{BMI}^{8}$. Measures of adipocyte lipolysis were available in a total of 939 study subjects (687 women, 252 men). Fifty-seven percent were obese (defined as $\mathrm{BMI} \geq 30 \mathrm{~kg} / \mathrm{m}^{2}$ ). 194 participants had type 2 diabetes, hypertension, or dyslipidemia alone or in different combinations.

\section{Ethics}

The study was approved by Forskningsetikkommittén vid Huddinge universitetssjukhus, that is the local committee on research ethics at Huddinge hospital (Sweden) (reference number 167/02) and all methods were performed in accordance with relevant guidelines and regulations. The study was explained in detail to each participant and informed consent was obtained from; this was in written form since 1996 when it became obligatory.

\section{Adipocyte lipolysis measurement in GENiAL}

Adipocyte lipolysis in clinical samples were measured as previously described ${ }^{5}$. Briefly, abdominal SAT biopsies obtained by needle aspiration were subjected to collagenase treatment to obtain isolated adipocytes. Fat cell suspensions (diluted to $2 \%$ volume/volume) were incubated for $2 \mathrm{~h}$ at $37^{\circ} \mathrm{C}$ with air as the gas phase in Krebs-Ringer phosphate buffer ( $\mathrm{pH} 7.4)$ supplemented with glucose $(8.6 \mathrm{mmol} / \mathrm{l})$, ascorbic acid $(0.1 \mathrm{mg} / \mathrm{ml})$, and bovine serum albumin $(20 \mathrm{mg} / \mathrm{ml})$ with the synthetic non-selective $\beta$ adrenoreceptor agonist isoprenaline (Hässle, Mölndal, Sweden) at concentrations $\left(10^{-9}-10^{-5} \mathrm{~mol} / \mathrm{l}\right.$; stimulated lipolysis) or without (spontaneous lipolysis). Quantity of glycerol as a measure of lipolysis was evaluated in an aliquot of medium at the end of the incubation ${ }^{999}(9)^{9}$. Spontaneous lipolysis was expressed as glycerol release/cell weight multiplied by the estimated abdominal subcutaneous fat mass, i.e., an estimate of the total release of glycerol from the area which is the site for the biopsy ${ }^{5}$. Herein we expressed isoprenaline-stimulated lipolysis as the quotient of glycerol release at the maximum effective isoprenaline concentration divided by the spontaneous rate (no hormone present) of glycerol release from the isolated fat cells. Values were $\log _{10}$ transformed to improve normality.

\section{Genetic analysis}

As previously described, the genetic analysis of spontaneous and stimulated lipolysis in GENiAL was performed in PLINK using linear regression, assuming an additive genetic model and including age, sex and BMI as covariates ${ }^{5}$. After quality control, 9,260,588 SNPs were analysed in 886 study subjects ${ }^{5}$. 
Mendelian randomisation analysis was conducted using mrbase (mrbase.org/pmid29846171) with default settings.

\section{Data mining}

In our analysis of BMI we used the SNPs listed in the file Meta-analysis_Locke_et_al + UKBiobank_2018_top_941_from_COJO_analysis_UPDATED which was reached by a link in Yengo et al. ${ }^{2}$ to https://portals.broadinstitute.org/collaboration/giant/index.php/GIANT_consortium_data_files and 2.2 BMI and Height GIANT and UK BioBank Meta-analysis Summary Statistics.

In our analysis of WHRadjBMI we used the SNPs listed in the file whradjbmi.giantukbb.meta.1.merged.indexSnps.combined.parsed which was reached by a link from Supplementary table 1 in Pulit et al. ${ }^{1}$ to https://github.com/lindgrengroup/fatdistnGWAS.

The FANTOM 5 dataset ${ }^{10}$ was used to assess gene expression in human adipose derived stem cells (hASCs) undergoing in vitro differentiation to adipocytes. Transcripts whose expression were $>10$ tags per million (TPM) at some point during the time course were defined as detected in hASCs.

Candidate genes were selected based on eQTL data in the GTEx ${ }^{11}$ (www.gtexportal.org) database and were retrieved in December 2019 and August 2020. All SNP-gene pairs listed in GTEx and reported herein reach FDR $<5 \%$. P-values for eQTL genes are nominal and taken from GTEx. There was no threshold for physical distance. Among SNP-gene pairs in Tables 1 and 2, four genes were located $>100 \mathrm{~Kb}$ from the SNP; only one gene was located at a distance > $500 \mathrm{Mb}$ from the SNP, EBPL ( $1 \mathrm{Mb}$ distance).

\section{Cell culture and transfection with small interfering RNA}

Isolation, growth, differentiation, and characterization of hASCs from a single donor have been described in detail previously in ${ }^{10,12}$. hASCs were transfected $24 \mathrm{~h}$ before induction of adipogenesis using ONTARGETplus SMARTpool small interfering RNAs (siRNAs) targeting candidate genes or control siRNA \#1 (Dharmacon/Horizon Discovery Group, Lafayette, CO, US) and Dharmafect 3 transfection agent (Dharmacon/Horizon Discovery Group) as previously described ${ }^{13}$. Differentiation to adipocytes was started by medium replacement 24 hours post-transfection and medium was subsequently replaced every third day. The RNA and medium were collected at days 2, 6 and 9 after the induction of differentiation and cells were fixed for lipid staining at day 9 .

For siNUP85 hASCs cells were also transfected $24 \mathrm{~h}$ postinduction of differentiation using a NEON electroporator (Invitrogen, Carlsbad, CA, US) according to the manufacturer's protocol. Briefly, for one transfection reaction 1 million hASCs suspended in $90 \mu$ l of R buffer was mixed with 200 pmol of siRNA targeting NUP85 or a control siRNA \#1 and transfection was performed using $100 \mu \mathrm{I}$ NEON electroporation tip. Electroporation conditions were $1600 \mathrm{~V}, 20 \mathrm{~ms}$ width, 1 pulse. Subsequently, cells were plated in antibiotic free medium at a density of 120000 cells/well in 24-well plates or 10000 cells/well in 96-well plates. Medium was replaced 24 hours post-transfection and subsequently every third day. For 
this type of experiment, the cells were cultured until day 3, 6 and 10 of differentiation, at which time RNA and medium were collected or cells were fixed for lipid staining.

Product identification numbers for siRNA are listed in Supplemental table 1 (ST 1).

\section{Measurement of lipolysis and lipid accumulation in in vitro differentiated adipocytes}

For glycerol measurements, medium was collected at day 9 of differentiation of hASCs for all tested genes and additionally at day 10 for siNUP85 when transfected at day 1 of differentiation. Glycerol is a valid end-product of lipolysis as it is re-utilized by fat cells to a negligible extent and was measured in cell culture medium as described ${ }^{14}$. Amounts of glycerol were normalized to the number of cells (evaluated as stained nuclei and described below) in each well.

Assessment of isoprenaline-stimulated lipolysis in siNUP85-transfected hASCs was performed as described previously ${ }^{15}$. In brief, cells were incubated for 3 hours in DMEM/F12 medium supplemented with $20 \mathrm{mg} / \mathrm{mL}$ of BSA and with or without isoprenaline $(1 \mu \mathrm{mol} / \mathrm{L})$. Glycerol in media was measured as described above. On collection of medium, cells from the same wells were lysed in RIPA buffer, protein concentration was measured, and glycerol levels were then corrected for total protein content.

Lipids were stained and quantified at differentiation day 9 for all the genes and additionally on day 10 for siNUP85 as previously described ${ }^{5}$. Briefly, following fixation with paraformaldehyde neutral lipids were stained with Bodipy 493/503 (0.2 $\mu \mathrm{g} / \mathrm{mL}$; Molecular Probes, Thermo Fisher Scientific, Waltham, MA, US) and nuclei (DNA) were stained with Hoechst 33342 (2 $\mu \mathrm{g} / \mathrm{mL}$; Molecular Probes) for $20 \mathrm{~min}$ at room temperature. Total Bodipy fluorescence (lipid droplets) was normalized to the number of nuclei (representing number of cells) in each well.

\section{RNA isolation and analysis of gene expression}

NucleoSpin RNA II kit (Macherey-Nagel, Düren, Germany) was used to extract total RNA from adipocytes. RNA concentration and quality were measured using Varioskan ${ }^{\text {TM }}$ LUX multimode microplate reader and $\mu$ Drop plate (ThermoFisher Scientific). Subsequently, $50 \mathrm{ng}$ of total RNA was reverse transcribed using the iScript cDNA synthesis kit (Bio-Rad, Hercules, CA, US). Quantitative RT-PCR was performed using 2.5 ng of cDNA and commercial TaqMan assays (Applied Biosystems/Thermo Fisher Scientific) in $10 \mu \mathrm{L}$ reaction on the CFX96 Touch $^{\text {TM }}$ qPCR Detection System (Bio-Rad) or QuantStudio Real-Time PCR (Thermo Fisher Scientific). Relative gene expression was calculated using the $2^{-\Delta C t}$ method ${ }^{16}$. Taqman assays are listed in ST1.

\section{Microarray assay and analysis}

Clariom $S$ arrays (Thermo Fisher Scientific) were used for global transcriptome profiling of primary adipocyte cultures treated with siRNA against ZNF436 $(n=6), N U P 85(n=6)$, or respective non-targeting siRNA control $(n=6)$ at day 6 of differentiation. The arrays were pre-processed in Transcriptome Analysis 
Console (Thermo Fisher Scientific) using SST-RMA, which includes normalization, summarization of probes to probesets, and background correction. We excluded 6,341 (ZNF436 samples) and 6,316 (NUP85 samples) probe sets with detection $p>0.05$ in at least one sample i.e., not detected in at least one sample, leaving 15,107 and 15,132 probe sets, respectively, for downstream analysis.

SAMR using block permutations was used to detect differentially expressed genes ${ }^{17}$. Webgestalt (www.webgestalt.org) was used to identify differentially expressed gene ontologies between siRNA treated and control samples.

\section{Statistical analysis}

Data from in vitro experiments were analysed by t-test. All p-values are two-tailed. A binomial test in Excel was used to analyse whether there was evidence that proportion of BMI and WHRadjBMI SNPs showing a directionally consistent effect on lipolysis was larger than the number expected by chance, i.e., $50 \%$.

When specific hypotheses are tested, a nominal $p<0.05$ is used as the significance threshold. In explorative transcriptome analysis multiple testing is taken into account and FDR $10 \%$ used as threshold.

\section{Results}

\section{Shared genetic loci for body fat storage and adipocyte lipolysis}

Data mining to identify shared genetic loci for $\mathrm{BMI}$ and adipocyte lipolysis is summarized in Fig. 1. A lookup of SNPs previously associated with BMI ${ }^{2}$ was conducted in the results of the GWAS of lipolysis ${ }^{5}$. Among 941 SNPs reported to be associated with BMI ${ }^{2}, 20$ displayed nominal association $(p<0.05)$ with spontaneous lipolysis with consistent effect directions for BMI and spontaneous lipolysis (ST 2). Five of these SNPs demonstrated expression quantitative trait loci (eQTLs) in SAT according to GTEx (Table 1). Furthermore, of 941 SNPs associated with BMI, 21 displayed nominal association with isoprenalinestimulated lipolysis with expected discordant effect direction for BMI and stimulated lipolysis (ST 3). Eight of the latter SNPs demonstrated eQTLs in SAT according to GTEx (Table 1).

We next assessed genetic influence on central fat distribution. Among index-SNPs in 346 genetic loci associated with WHRadjBMI ${ }^{1}, 12$ displayed nominal associations $(p<0.05)$ with spontaneous lipolysis; eight of these SNPs demonstrated eQTLs in SAT according to GTEx (Table 2, ST 4). 20 SNPs displayed associations with WHRadjBMI and isoprenaline-stimulated lipolysis (ST 5) of which ten SNPs were eQTLs in SAT (Table 2). In the case of WHRadjBMI, effect directions were not considered because there is limited information regarding the relationship between lipolysis in different adipose depots and adipose distribution. None of the SNPs mentioned herein are among SNPs previously reported in the GENiAL cohort to display GWAS suggestive association with lipolysis ${ }^{5}$.

\section{Functional evaluation of candidate genes in shared BMI- lipolysis loci}


Three eQTL genes (ZNF436, WARS2, TBX15) in the shared BMI-spontaneous lipolysis loci and five eQTL genes (ZNF436, FLJ20021, INVS, STX17, ALDH2) in the shared BMI-stimulated lipolysis loci were expressed in hASCs (Table 1, Supplemental Fig. 1a). Of these, five genes (ZNF436, WARS2, TBX15, INVS and STX17) were taken forward for functional siRNA screen in hASCs. FLJ20021 was excluded as, until recently, it has been annotated as an uncharacterized IncRNA ${ }^{18}$. ALDH2 was excluded because it has recently been established as a positive regulator of adipocyte differentiation ${ }^{19}$. We knocked-down the five candidate genes prior to induction of differentiation, followed by evaluation of glycerol levels in conditional medium as a marker of lipolysis. Knock-down of ZNF436, TBX15 and STX1, but not WARS2 and INVS, decreased glycerol levels (Fig. 2a). To assess whether the impact on lipolysis was direct or secondary to disturbances in adipogenesis, we stained the cells for neutral lipid content. Knock-down of $T B X 15$, but not the other investigated genes, decreased amount of lipids (Fig. 2b). Results for the BMIpanel genes ZNF436 and STX17 were of particular interest because knock-down of these two genes affected glycerol release without affecting lipid content supporting the hypothesis that the impact on lipolysis was direct, and not indirect via influence on adipogenesis.

In parallel, we evaluated the effect of knock-down efficiency of the studied genes at the mRNA level. In general, knock-down efficiency was good, i.e., varied between $90-70 \%$ with exception of TBX15 where knock-down efficiency reached only $50 \%$ at day 6 (Fig. 3a-b and Supplemental Fig. 2a-c). For the genes affecting glycerol we evaluated whether gene knock-down effects could be linked to altered expression levels of key genes such as LIPE and PNPLA2 encoding lipases, PLIN1 encoding the lipid coating protein Perilipin 1, or expression of $A P I D O Q$ encoding the adipocyte marker adiponectin. Knock-down of ZNF436 caused temporary downregulation of PLIN1, PNPLA2 and ADIPOQ (Fig. 3a). Knock-down of STX17 upregulated $\angle I P E$ and $A D I P O Q$ at both day 6 and 9 of differentiation, while upregulation of $P L I N 1$ and PNPLA2 was temporal (Fig. 3b). Knock-down of TBX15 had minor effects on studied gene expression (Supplemental Fig. 2b).

\section{Functional evaluation of candidate genes in shared WHRadjBMI-lipolysis loci}

We next selected eQTL genes in the shared WHRadjBMI-lipolysis loci for functional evaluation in vitro. Four genes in the shared WHRadjBMI-spontaneous lipolysis loci were expressed in hASCs: TBX15, WARS2 (both included in analysis of BMI-loci above), ZNF263 and PCK1 (Table 2). Seven genes in the shared WHRajdBMI-stimulated lipolysis loci were expressed in hASCs: EBPL, NID2, GGA3, MRPS7, NUP85, GRB2, and PEMT (Table 2, Supplemental Fig. 1b). Knock-down of PCK1, NID2, GGA3, NUP85 and GRB2 decreased levels of glycerol by $20-70 \%$ (Fig. 2c). Knock-down of EBPL, MRPS7 and PEMT had no effect (Fig. 2c). Among the genes controlling glycerol release, accumulation of lipids was decreased by knockdown of PCK1, NID2 and GRB2. (Fig. 2e). Knock down of ZNF263 showed no effect on glycerol or lipid levels most likely due to due to poor knockdown in the tested conditions (data not shown).

The inhibition of spontaneous lipolysis by PCK 1 knock-down was not consistent with the genetic data, where the $T$ allele is associated with high gene expression and low spontaneous lipolysis, and this gene 
was therefore irrelevant for further studies. NUP85 and GGA3 were the only two genes from the WHRadjBMI panel that affected glycerol release without affecting lipid content. However, knocking down GGA3 at different time points during differentiation decreased lipid accumulation (data not shown). Based on this and its effect on key adipocyte genes (Fig. 3c, also see below) we could not rule out an effect of adipogenesis. Thus, NUP85 was the only remaining gene from the WHRadjBMI panel that affected glycerol accumulation without affecting lipid content supporting that the impact on lipolysis was direct, and not indirect via influence on adipogenesis. Since the NUP85 SNP rs9909443 was associated with isoprenaline-stimulated lipolysis (Table 2), we also evaluated effects of NUP85 knockdown on isoprenaline-stimulated lipolysis, which was decreased compared to the control siRNA (Fig. 2d). In this experiment in basal condition (no isoprenaline) we did not detect lower glycerol release in siNUP85 samples compared to control siRNA. However, this is only a $3 \mathrm{~h}$ "window" of lipolysis and the accumulative effect seems to be important to obtain effects on glycerol release by NUP85.

As for BMI genes, we evaluated knock-down efficiency of WHRadjBMI genes and assessed whether expression of known lipolysis genes could be affected. Knock-down efficiency varied from 75 to $95 \%$ (Supplemental Fig. 2e-i) except for PCK1 and GGA3 (Supplemental Fig. 2d and Fig. 3c). These genes were very low expressed in the beginning of differentiation and therefore it is possible that the siRNAtransfection conditions need to be further optimized. Furthermore, knock-down of WHRadjBMI-associated genes had less effect on adipocyte gene expression than BMI-associated genes. Knock-down of PCK1 upregulated $\angle I P E$ and temporally downregulated PNPLA2 and ADIPOQ (Supplemental Fig. 2d). Knockdown of GGA3, NID2 and GRB2 temporary downregulated all studied genes (Fig. 3c and Supplemental Fig. 2f, h) while knock-down of NUP85 had no effect (Fig. 3d).

\section{ZNF436 and NUP85 target genes}

The analysis of expression of central genes controlling lipolysis (LIPE, PLIN1, and PNPLA2) did not reveal a functional link to lipolysis for the shared GWAS-candidate genes for BMI/WHRadjBMI and lipolysis. Therefore, two genes were taken forward for global transcriptome analysis: ZNF436 from the BMI and NUP85 from the WHRajdBMI analysis. They were selected because the genetic, metabolic, and functional results for lipolysis were consistent, e.g., rs967605-C was associated with high ZNF436 expression, BMI and spontaneous lipolysis, and ZNF436 knock-down inhibited glycerol release. Furthermore, for both genes it was reasonable to assume that the impact on lipolysis was indirect via influence in gene expression; ZNF436 is a transcription factor ${ }^{20}$ and NUP85 encodes a protein forming part of nuclear pores, which in turn are involved in transcriptional regulation ${ }^{21-23}$.

Transcriptome analysis revealed 396 genes that were differentially expressed between ZNF436 knockdown and control cells with FDR $<10 \%$, of which 203 were downregulated n (ST 6). Downregulated genes were enriched for the gene ontology (GO) "regulation of lipid metabolic process" (ST 7). Expression of three genes ( $P N P L A 3, P D E 3 B$ and $M G L L$ ) reported to be involved in lipolysis ${ }^{24}$ were among $Z N F 436$ regulated genes (ST 6, Supplemental Fig. 3). 
336 genes were differentially expressed between NUP85 knockdown and control cells with FDR $<10 \%$, of which only five were upregulated (ST 8). Downregulated GO included "extracellular structure organization" and "regulation of lipid metabolic process" (ST 9). Expression of two genes reported to be involved in lipolysis were among NUP85-regulated genes [NPR3 fold change (f.c.) 0.70, ADRB1 f.c. 0.77] (ST 8, Supplemental Fig. 3).

\section{Mendelian Randomisation analysis}

To assess relationship between BMI and WHRadjBMI and lipolysis ee used Mendelian Randomisation analysis. We provide evidence of causal effects of spontaneous and stimulated lipolysis on BMI but not WHRadjBMI (ST 10, Supplemental Figs. 4-5). These results should be interpreted with caution, as the selection of SNPs for mendelian randomisation analyses was non-standard, i.e., the SNPs were not GWAS significant. Whilst the results for spontaneous lipolysis are convincing, those for stimulated lipolysis are curious, as the MR Egger is null, despite the lack of horizontal pleiotropy and the NOME assumption being fulfilled (IGX2 =1). We have also analysed directionally consistency for all BMI and WHRadjBMI GWAS SNPs for association with lipolysis in GENiAL. Results were significant for BMI but not WHRadjBMI (ST 11).

\section{Discussion}

The findings reported here enhance our understanding of how genetic variants mediate their impact on the complex traits of fat storage and distribution. In the shared lipolysis-BMI or lipolysis-WHRadjBMI associated SNP panels we identified five and seven SNPs, respectively, displaying allele dependent gene expression for genes expressed in fat cells. Among these genes, we provided functional evidence supporting the hypothesis that transcriptional regulation of ZNF436 and NUP85 have intrinsic effects on lipolysis consistent with the associations in the clinical cohort and propose that these genes hereby are involved in the genetic regulation of BMI and WHRadjBMI (Fig. 4). Furthermore, candidate genes in one more BMI-locus (STX17) and four more WHRadjBMI loci (PCK1, NID2, GGA3 and GRB2) control lipolysis alone, or in conjunction with lipid storage, and may hereby be involved in body fat storage.

In the BMI-associated locus on chromosome 1 we showed that rs $967605-\mathrm{C}$ is associated with higher $\mathrm{BMI}$, adipocyte spontaneous lipolysis, and ZNF436 expression. Consistent with these associations found in clinical cohorts knock-down of ZNF436 inhibited adipocyte lipolysis, whereas no impact on lipid accumulation was observed. ZNF436 is a member of the zinc finger transcription factor family and may act as a negative regulator on gene transcription mediated by MAPK signaling ${ }^{20}$. ZNF436 mRNA is enriched in adipocytes as compared to cells from other organs ${ }^{10}$. ZNF436 knock-down followed by transcriptome analysis revealed that ZNF436 acts as a regulator of many genes involved in lipid metabolic processes. However, ZNF436 did not control lipolysis via transcriptional regulation of hitherto described genes in the canonical human lipolysis process ${ }^{24,25}$. Importantly, the expression of a set of 
other genes reported to influence lipolysis or related processes, outside the canonical lipolysis pathway, was regulated by ZNF436 knock-down (ST 6), e.g.:

- QRFP encoding the neuropeptide 26RFa, which controls phosphorylation of HSL and PLIN1 and hereby inhibits lipolysis ${ }^{26}$.

- PDE4A encoding one subtype of phosphodiesterase 4, which inhibits lipolysis ${ }^{27}$.

- ARHGAP26 which is involved in endocytosis and inhibits lipolysis ${ }^{28}$.

- TRAF2 encoding TNF receptor-associated factor 2, which stimulates TNFA-induced lipolysis ${ }^{29}$.

- TTC39B encoding a scaffolding protein that promotes ubiquitination and degradation of liver $\mathrm{X}$ receptor, a transcription factor that in turn stimulates lipolysis. In addition, TTC39B antisense treatment protects against obesity in mice ${ }^{30}$.

On chromosome 9 rs10118701-G was associated with higher BMI, STX17 expression, and lower stimulated lipolysis. Knock-down of STX17 inhibited spontaneous lipolysis. We did not follow up these findings to determine whether the gene had an opposing inhibitory effect on stimulated as compared to spontaneous lipolysis consistent with the opposing genetic association in the clinical cohort. One example of a gene with such opposing effects is PLIN1, which suppresses spontaneous and facilitates stimulated lipolysis ${ }^{31}$. STX17 encodes a key component of the autophagosome, which in turn has been functionally linked to lipolysis ${ }^{32}$.

In the WHRadjBMI-associated locus on chromosome 17 we showed that rs9909443-T is associated with higher stimulated lipolysis and NUP85 gene expression, as well as lower WHRadjBMI. NUP85 knock-down resulted in consistent inhibition of spontaneous and stimulated lipolysis without affecting lipids giving support to the hypothesis that NUP85, rather than other genes is this locus, is causally linked to lipolysis. Central fat accumulation has been associated with reduced stimulated lipolysis ${ }^{33}$, but the relationship between lipolysis and fat distribution is less straight forward for fat distribution than for BMI since there are depot specific differences in gene expression and fat cell metabolism between e.g. abdominal and femoral fat depots ${ }^{34}$. NUP85 encompasses an essential component of the nuclear pore complex important for nuclear transport but has also been reported to be involved in DNA replication and gene activation ${ }^{35}$. The inhibitory effect of NUP85 knock-down on lipolysis was accompanied by downregulated expression of numerous genes. Potentially, downregulated expression of ADRB1 could contribute to inhibition of stimulated lipolysis. Other NUP85 regulated genes may mediate the effects of NUP85 deficiency on lipolysis e.g.:

- BMPR2 deficiency in adipocytes inhibits phosphorylation of the lipid-droplet-coating protein perilipin ${ }^{36}$ and hereby impairs stimulated lipolysis. 
- EIF4EBP1. Simultaneous lack of EIF4EBP1 and EIF4EBP2 increases sensitivity to diet-induced obesity and insulin resistance in mice ${ }^{37}$ and is involved in the regulation of the lipid-droplet-coating protein ATGL 38 .

- DDIT4 is a fasting-regulated p53 target gene. Forced expression of DDIT4 augment lipolysis in adipocytes ${ }^{39}$. In summary, the herein reported regulation of lipolysis by NUP85 describes a new role for this gene in adipose biology.

Further analysis of WHRadjBMI loci revealed that at the chromosomes 14 and 17 knock-down of NID2, $G R B 2$, and $G G A 3$, respectively, inhibited both glycerol release and lipid accumulation. The simultaneous effects of these genes on lipid accumulation and on glycerol release prevented us from drawing any conclusion as regards which metabolic processes link SNPs in these loci to body fat distribution. In the joint BMI-WHRadjBMI locus on chromosome 1 the inhibition of spontaneous lipolysis by TBX15 knockdown was not consistent with the genetic data where the T allele was associated with high TBX15 gene expression and low spontaneous lipolysis. It is therefore likely that other functions in adipose tissue linked to TBX15 may mediate the impact of this locus of body fat storage ${ }^{40}$.

One limitation of the study is that no adjustment for multiple testing was used when selecting SNPs associated with lipolysis for functional follow up. The approach was justified by strong prior biological knowledge of the relationships between obesity and the cellular phenotype in question. We have tried to balance the risk of false-positives and negatives results by using a p-value threshold and combined this with a strong and clear biological rationale, that is the importance of lipolysis in the function of adipose tissue. Importantly, a causal relationship between lipolysis and BMI was supported by both Mendelian randomization analysis and overall enrichment of SNPs displaying directionally consistent effects on $\mathrm{BMI}$ and lipolysis. The lack of such evidence for WHRadjBMI may be related to limited power of the study and a more complex association between lipolysis in different fat depots and adipose distribution.

Another limitation is that we only looked for eQTL genes retrieved from the GTEx portal which only reports genetic effects on gene expression in tissue samples and not those limited to adipocytes. Thus, we may not have identified all BMI/WHRadjBMI-SNPs controlling gene function in adipocytes. Furthermore, among the genetic loci with genes controlling lipolysis in siRNA experiments, only rs146182298 (NID2) and rs1328757 (PCK1) were potential lead eQTL SNPs, i.e., they were among the SNPs most strongly associated with expression of the specific gene according to visual inspection in GTEx. A third limitation is that we only assessed lipolysis in abdominal SAT although other adipose depots might be more relevant ${ }^{41}$, but are even less accessible for sampling in adequate numbers.

In summary, our study highlights that genetic susceptibility to central obesity might be associated with risk of impaired adipose lipolysis. We provided functional evidence supporting the notion that transcriptional regulation of ZNF436, and NUP85 have intrinsic effects on lipolysis consistent with the associations in the clinical cohort and propose that these genes hereby are involved in the genetic regulation of BMI and WHRadjBMI (Fig. 4). 


\section{Declarations}

\section{Acknowledgements}

Authors thank all participants and staff involved in the GENiAL study. The excellent technical assistance of Ana Maria Suzuki, Thais de Castro Barbosa, Gaby Åström, Elisabeth Dungner, Kerstin Wåhlen, Eva Sjölin, Katarina Hertel and Yvonne Widlund is highly appreciated. We thank Marika Rönnholm for excellent technical assistance with the gene microarray, and David Brodin for array processing. We thank Keira J.A. Johnston for her assistance in interpreting the MR analyses.

\section{Author Contributions}

AK designed the study, performed literature search, conducted analysis, interpreted the data, conducted experiments, created figures and tables, drafted, and edited the manuscript; VL conducted experiments, interpreted the data and edited the manuscript; RJS conducted analysis, performed literature search, created figures, interpreted the data and edited the manuscript; PA acquired the data, conceived and designed the study, interpreted the data, and edited the manuscript. ID acquired the data, conceived and designed the study, conducted analysis, interpreted the data, performed literature search, created figures and tables, drafted and edited the manuscript. All authors have agreed to be accountable for their contributions and have approved the final manuscript.

\section{Funding Sources}

RJS is supported by a the UKRI innovation-HDR-UK Fellowship (MR/S003061/1). ID is supported by the Strategic Research Program in Diabetes at Karolinska Institutet (Genetic and long-term epigenetic studies of changes in adipose function), Swedish Research Council (2019-00997), Novo Nordisk foundation (NNF200C0063582), and the Swedish Diabetes Association (DIA2019-407). PA is supported by the Strategic Research Program in Diabetes at Karolinska Institutet for studies of human fat cell function. The funders had no role in study design; in the collection, analysis, and interpretation of data; in the writing of the manuscript; or in the decision to submit the paper for publication.

\section{Conflict of Interest}

None.

\section{Data sharing}

Summary statistics from the GWAS analysis of spontaneous and isoprenaline-stimulated lipolysis, experimental data and gene array data are available upon request (ingrid.dahlman@ki.se).

\section{References}


1. Pulit, S. L. et al. Meta-analysis of genome-wide association studies for body fat distribution in 694 649 individuals of European ancestry. Human molecular genetics, 28, 166-174 https://doi.org/10.1093/hmg/ddy327 (2019).

2. Yengo, L. et al. Meta-analysis of genome-wide association studies for height and body mass index in 700000 individuals of European ancestry. Human molecular genetics, 27, 3641-3649 https://doi.org/10.1093/hmg/ddy271 (2018).

3. Arner, P., Andersson, D. P., Bäckdahl, J., Dahlman, I. \& Rydén, M. Weight Gain and Impaired Glucose Metabolism in Women Are Predicted by Inefficient Subcutaneous Fat Cell Lipolysis. Cell metabolism, 28, 45-5443 https://doi.org/10.1016/j.cmet.2018.05.004 (2018).

4. Arner, P. \& Langin, D. Lipolysis in lipid turnover, cancer cachexia, and obesity-induced insulin resistance. Trends in endocrinology and metabolism: TEM, 25, 255-262 https://doi.org/10.1016/j.tem.2014.03.002 (2014).

5. Kulyté, A. et al. Genome-wide association study of adipocyte lipolysis in the GENetics of adipocyte lipolysis (GENiAL) cohort. Molecular metabolism, 34, 85-96 https://doi.org/10.1016/j.molmet.2020.01.009 (2020).

6. Locke, A. E. et al. Genetic studies of body mass index yield new insights for obesity biology. Nature, 518, 197-206 https://doi.org/10.1038/nature14177 (2015).

7. Shungin, D. et al. New genetic loci link adipose and insulin biology to body fat distribution. Nature, 518, 187-196 https://doi.org/10.1038/nature14132 (2015).

8. Heid, I. M. et al. Meta-analysis identifies 13 new loci associated with waist-hip ratio and reveals sexual dimorphism in the genetic basis of fat distribution. Nature genetics, 42, 949-960 https://doi.org/10.1038/ng.685 (2010).

9. Hellmér, J., Arner, P. \& Lundin, A. Automatic luminometric kinetic assay of glycerol for lipolysis studies. Analytical biochemistry, 177, 132-137 https://doi.org/10.1016/0003-2697(89)90027-4 (1989).

10. Arner, E. et al. Transcribed enhancers lead waves of coordinated transcription in transitioning mammalian cells. Science (New York, N. Y.), 347, 1010-1014 https://doi.org/10.1126/science.1259418 (2015).

11. Consortium, G. T. The Genotype-Tissue Expression (GTEx) project. Nature genetics, 45, 580-585 https://doi.org/10.1038/ng.2653 (2013).

12. Pettersson, A. M. et al. LXR is a negative regulator of glucose uptake in human adipocytes., 56 , 2044-2054 https://doi.org/10.1007/s00125-013-2954-5 (2013).

13. Jiao, H. et al. Whole-Exome Sequencing Suggests LAMB3 as a Susceptibility Gene for Morbid Obesity., 65, 2980-2989 https://doi.org/10.2337/db16-0522 (2016).

14. Lundbäck, V. et al. FAM13A and POM121C are candidate genes for fasting insulin: functional followup analysis of a genome-wide association study., 61, 1112-1123 https://doi.org/10.1007/s00125018-4572-8 (2018). 
15. Arner, P. et al. Mapping of biguanide transporters in human fat cells and their impact on lipolysis. Diabetes Obes Metab, 20, 2416-2425 https://doi.org/10.1111/dom.13395 (2018).

16. Livak, K. J. \& Schmittgen, T. D. Analysis of relative gene expression data using real-time quantitative PCR and the 2(-Delta Delta C(T)) Method., 25, 402-408 https://doi.org/10.1006/meth.2001.1262 (2001).

17. Tusher, V. G., Tibshirani, R. \& Chu, G. Significance analysis of microarrays applied to the ionizing radiation response. Proceedings of the National Academy of Sciences of the United States of America, 98, 5116-5121 https://doi.org/10.1073/pnas.091062498 (2001).

18. Meng, N. et al. Small Protein Hidden in IncRNA LOC90024 Promotes "Cancerous" RNA Splicing and Tumorigenesis. Advanced science (Weinheim. Baden-Wurttemberg, Germany), 7, 1903233 https://doi.org/10.1002/advs.201903233 (2020).

19. Yu, Y. H. et al. PKC-ALDH2 Pathway Plays a Novel Role in Adipocyte Differentiation. PloS one, 11, e0161993 https://doi.org/10.1371/journal.pone.0161993 (2016).

20. Li, Y. et al. A novel zinc-finger protein ZNF436 suppresses transcriptional activities of AP-1 and SRE. Mol Biol Rep, 33, 287-294 https://doi.org/10.1007/s11033-006-9019-5 (2006).

21. D'Angelo, M. A. Nuclear pore complexes as hubs for gene regulation. Nucleus, 9, 142-148 https://doi.org/10.1080/19491034.2017.1395542 (2018).

22. Ibarra, A. \& Hetzer, M. W. Nuclear pore proteins and the control of genome functions. Genes Dev, 29, 337-349 https://doi.org/10.1101/gad.256495.114 (2015).

23. Pascual-Garcia, P. \& Capelson, M. Nuclear pores as versatile platforms for gene regulation. Curr Opin Genet Dev, 25, 110-117 https://doi.org/10.1016/j.gde.2013.12.009 (2014).

24. Stenson, B. M. et al. Liver $X$ receptor (LXR) regulates human adipocyte lipolysis. J Biol Chem, 286, 370-379 https://doi.org/10.1074/jbc.M110.179499 (2011).

25. Yang, A., Mottillo, E. P., Mladenovic-Lucas, L., Zhou, L. \& Granneman, J. G. Dynamic interactions of ABHD5 with PNPLA3 regulate triacylglycerol metabolism in brown adipocytes. Nat Metab, 1, 560569 https://doi.org/10.1038/s42255-019-0066-3 (2019).

26. Mulumba, M., Granata, R., Marleau, S. \& Ong, H. QRFP-43 inhibits lipolysis by preventing ligandinduced complex formation between perilipin A, caveolin-1, the catalytic subunit of protein kinase and hormone-sensitive lipase in 3T3-L1 adipocytes. Biochimica et biophysica acta, 1851, 657-666 https://doi.org/10.1016/j.bbalip.2015.02.005 (2015).

27. Wang, H. \& Edens, N. K. mRNA expression and antilipolytic role of phosphodiesterase 4 in rat adipocytes in vitro. Journal of lipid research, 48, 1099-1107 https://doi.org/10.1194/jlr.M600519JLR200 (2007).

28. Lucken-Ardjomande Hasler, S., Vallis, Y., Jolin, H. E., McKenzie, A. N. \& McMahon, H. T. GRAF1a is a brain-specific protein that promotes lipid droplet clustering and growth, and is enriched at lipid droplet junctions. Journal of cell science, 127, 4602-4619 https://doi.org/10.1242/jcs.147694 (2014). 
29. Kawamata, Y. et al. Tumor necrosis factor receptor-1 can function through a $\mathrm{G}$ alpha q/11-betaarrestin-1 signaling complex. J Biol Chem, 282, 28549-28556 https://doi.org/10.1074/jbc.M705869200 (2007).

30. McCabe, K. M. et al. Antisense oligonucleotide treatment produces a type I interferon response that protects against diet-induced obesity. Molecular metabolism, 34, 146-156 https://doi.org/10.1016/j.molmet.2020.01.010 (2020).

31. Brasaemle, D. L. Thematic review series: adipocyte biology. The perilipin family of structural lipid droplet proteins: stabilization of lipid droplets and control of lipolysis. Journal of lipid research, 48, 2547-2559 https://doi.org/10.1194/jlr.R700014-JLR200 (2007).

32. Maeda, Y., Oku, M. \& Sakai, Y. A defect of the vacuolar putative lipase Atg15 accelerates degradation of lipid droplets through lipolysis. Autophagy, 11, 1247-1258 https://doi.org/10.1080/15548627.2015.1056969 (2015).

33. Dahlman, I. et al. Numerous Genes in Loci Associated With Body Fat Distribution Are Linked to Adipose Function., 65, 433-437 https://doi.org/10.2337/db15-0828 (2016).

34. Denton, N. F. et al. Bone morphogenetic protein 2 is a depot-specific regulator of human adipogenesis. Int J Obes (Lond), 43, 2458-2468 https://doi.org/10.1038/s41366-019-0421-1 (2019).

35. Harel, A. et al. Removal of a single pore subcomplex results in vertebrate nuclei devoid of nuclear pores. Mol Cell, 11, 853-864 https://doi.org/10.1016/s1097-2765(03)00116-3 (2003).

36. Qian, S. et al. BMPR2 promotes fatty acid oxidation and protects white adipocytes from cell death in mice. Commun Biol, 3, 200 https://doi.org/10.1038/s42003-020-0928-y (2020).

37. Le Bacquer, $\mathrm{O}$. et al. Elevated sensitivity to diet-induced obesity and insulin resistance in mice lacking 4E-BP1 and 4E-BP2. J Clin Invest, 117, 387-396 https://doi.org/10.1172/JCl29528 (2007).

38. Singh, M. et al. 4E-BPs Control Fat Storage by Regulating the Expression of Egr1 and ATGL. J Biol Chem, 290, 17331-17338 https://doi.org/10.1074/jbc.M114.631895 (2015).

39. Schupp, M. et al. Metabolite and transcriptome analysis during fasting suggest a role for the p53Ddit4 axis in major metabolic tissues. BMC Genomics, 14, 758 https://doi.org/10.1186/1471-216414-758 (2013).

40. Gesta, S. et al. Mesodermal developmental gene Tbx15 impairs adipocyte differentiation and mitochondrial respiration. Proceedings of the National Academy of Sciences of the United States of America, 108, 2771-2776 https://doi.org/10.1073/pnas.1019704108 (2011).

41. Lotta, L. A. et al. Integrative genomic analysis implicates limited peripheral adipose storage capacity in the pathogenesis of human insulin resistance. Nature genetics, $49,17-26$ https://doi.org/10.1038/ng.3714 (2017).

\section{Tables}

Table 1. eQTL SNPs displaying congruent association with adipocyte lipolysis and BMI 


\begin{tabular}{|c|c|c|c|c|c|c|c|c|c|c|c|c|c|c|c|c|c|}
\hline \multirow[b]{2}{*}{ \$NP } & \multirow[b]{2}{*}{ Chrom. } & \multirow[b]{2}{*}{ Pos. } & \multicolumn{5}{|c|}{ Spontaneous lipolysis } & \multicolumn{5}{|c|}{ BMI } & \multicolumn{4}{|c|}{ eQTL (SAT) } & \multirow{2}{*}{$\begin{array}{l}\text { Detected } \\
\text { in hASCs }\end{array}$} \\
\hline & & & Beta & $P$ & Ref & Alt & Freq & Beta & $P$ & Ref & Alt & Freq & Gene & $P$ & High & Low & \\
\hline \multirow[t]{2}{*}{ rs967605 } & 1 & 23399932 & 0.07 & $7.78 \mathrm{E}-03$ & $\mathrm{~T}$ & $\mathrm{C}$ & 0.14 & -0.02 & $3.68 \mathrm{E}-13$ & $\mathrm{C}$ & $\mathrm{T}$ & 0.83 & ZNF436 & 0.0000086 & $\mathrm{C}$ & $\mathrm{T}$ & \\
\hline & & & & & & & & & & & & & ZNF 430-ASI & 0.000018 & $\mathrm{C}$ & $\mathrm{T}$ & n.d. \\
\hline rs10923724 & & & & & & & & & & & & & TBX15 & 0.0000097 & $\mathrm{~T}$ & $\mathrm{C}$ & \\
\hline rs919433 & 2 & 198166565 & -0.04 & $2.67 \mathrm{E}-02$ & A & G & 0.62 & 0.01 & $1.36 \mathrm{E}-11$ & G & A & 0.36 & ANKRD44 & $7.30 \mathrm{E}-13$ & G & A & n.d. \\
\hline rs4515655 & 9 & 128616073 & -0.04 & 4.37E-02 & $\mathrm{C}$ & $\mathrm{T}$ & 0.38 & -0.01 & $1.01 \mathrm{E}-09$ & $\mathrm{C}$ & $\mathrm{T}$ & 0.40 & PBX3 & $3.10 \mathrm{E}-17$ & $\mathrm{~T}$ & $\mathrm{C}$ & n.d. \\
\hline \multirow[t]{2}{*}{ rs17105272 } & 14 & 77529783 & -0.04 & $4.15 \mathrm{E}-02$ & $\mathrm{~T}$ & C & 0.67 & 0.01 & $2.22 \mathrm{E}-09$ & $\mathrm{C}$ & $\mathrm{T}$ & 0.32 & RP11-7F17.3 & $4.20 \mathrm{E}-09$ & $\mathrm{~T}$ & $\mathrm{C}$ & n.d. \\
\hline & & & \multicolumn{6}{|c|}{ Isoprenaline stimulated lipolysis } & \multicolumn{3}{|c|}{ BMI } & & \multicolumn{4}{|c|}{ eQTL (SAT) } & Detected \\
\hline SNP & Chrom. & Pos. & Beta & $P$ & Ref & Alt & Freq & Beta & $P$ & Ref & Alt & Freq & GENE & $P$ & High & Low & in hASCs \\
\hline \multirow[t]{2}{*}{ rs967605 } & 1 & 23399932 & -0.06 & $1.63 \mathrm{E}-03$ & $\mathrm{~T}$ & $\mathrm{C}$ & 0.14 & -0.02 & $3.68 \mathrm{E}-13$ & $\mathrm{C}$ & $\mathrm{T}$ & 0.83 & ZNF436 & $8.60 \mathrm{E}-06$ & $\mathrm{C}$ & $\mathrm{T}$ & \\
\hline & & & & & & & & & & & & & $Z N F 436-A S 1$ & $1.80 \mathrm{E}-05$ & $\mathrm{C}$ & $\mathrm{T}$ & n.d. \\
\hline rs2850969 & 4 & 102183594 & 0.04 & $2.81 \mathrm{E}-02$ & $\mathrm{C}$ & $\mathrm{T}$ & 0.85 & -0.01 & $2.16 \mathrm{E}-09$ & $\mathrm{C}$ & $\mathrm{T}$ & 0.86 & FLJ20021 & $1.90 \mathrm{E}-11$ & $\mathrm{~T}$ & $\mathrm{C}$ & \\
\hline rs450231 & 9 & 101481205 & -0.03 & $2.76 \mathrm{E}-02$ & A & G & 0.29 & -0.01 & $2.59 \mathrm{E}-12$ & G & A & 0.75 & ANKSO & 0.000065 & G & A & n.d. \\
\hline rs10118701 & 9 & 103061366 & 0.04 & $1.02 \mathrm{E}-02$ & G & A & 0.66 & -0.01 & $3.46 \mathrm{E}-12$ & G & A & 0.68 & INVS & 1.70 E-13 & G & A & \\
\hline rs 4515655 & 9 & 128616073 & 0.03 & $4.34 \mathrm{E}-02$ & $\mathrm{C}$ & $\mathrm{T}$ & 0.38 & -0.01 & 1.01 E-09 & $\mathrm{C}$ & $\mathrm{T}$ & 0.40 & $P B X 3$ & 3.10 E- 17 & $\mathrm{~T}$ & $\mathrm{C}$ & n.d. \\
\hline \multirow[t]{2}{*}{ rs3781099 } & 10 & 27318776 & 0.05 & 4.67E-02 & $\mathrm{T}$ & $\mathrm{C}$ & 0.92 & 0.02 & $8.07 \mathrm{E}-11$ & $\mathrm{C}$ & $\mathrm{T}$ & 0.08 & MASTL & 4.20E-11 & $\mathrm{C}$ & $\mathrm{T}$ & n.d. \\
\hline & & & & & & & & & & & & & $L I N C 00202-1$ & 0.0000022 & $\mathrm{~T}$ & $\mathrm{C}$ & n.d. \\
\hline rs 1558236 & 12 & 111780998 & -0.09 & $2.09 \mathrm{E}-02$ & G & $\mathrm{C}$ & 0.97 & 0.06 & $9.85 \mathrm{E}-15$ & G & $\mathrm{C}$ & 0.97 & $A L D H 2$ & 0.0000018 & $G$ (rare) & $\mathrm{C}$ & \\
\hline rs 2537847 & 17 & 65694355 & 0.03 & $3.14 \mathrm{E}-02$ & $\mathrm{G}$ & A & 0.25 & -0.01 & $3.39 \mathrm{E}-09$ & $\mathrm{G}$ & A & 0.24 & RP11-855A2.1 & 0.000071 & A & $\mathrm{G}$ & n.d. \\
\hline
\end{tabular}

Where Chrom. $=$ chromosome, Pos. = genome position in basepairs accoding to GRCh37, Beta refer to Alt allele, Freq $=$ frequency of Alt allele,

High = allele with highest gene expression according to GTEx; n.d. = mRNA not detected in hASC undergoing in vitro differentiation to adipocytes.

Table 2. eQTL SNPs associated with adipocyte lipolysis and WHRadjBMI

\begin{tabular}{|c|c|c|c|c|c|c|c|c|c|c|c|c|c|c|c|}
\hline \multirow[b]{2}{*}{ SNP } & \multirow[b]{2}{*}{ Chrom. } & \multirow[b]{2}{*}{ Pos. } & \multicolumn{5}{|c|}{ Spontaneous lipolysis } & \multicolumn{4}{|c|}{ WHRadjBMII } & \multicolumn{3}{|c|}{ eQTL (SAT) } & \multirow{2}{*}{$\begin{array}{l}\text { Detected } \\
\text { in hASCs }\end{array}$} \\
\hline & & & Beta & $P$ & Ref & Alt & Freq & Beta & Ref & Alt & Freq & Gene & High & Low & \\
\hline \multirow[t]{2}{*}{ rs 10923724} & 1 & 119546842 & 0.05 & $8.33 \mathrm{E}-03$ & $\mathrm{~T}$ & $\mathrm{C}$ & 0.40 & 0.03 & $\mathrm{C}$ & $\mathrm{T}$ & 0.56 & WARS2 & $\mathrm{C}$ & $\mathrm{T}$ & \\
\hline & & & & & & & & & & & & TBXIS & $\mathrm{T}$ & $\mathrm{c}$ & \\
\hline \multirow[t]{2}{*}{ rs10055995 } & 5 & 137698299 & -0.06 & $5.17 \mathrm{E}-04$ & $\mathrm{~T}$ & $\mathrm{c}$ & 0.42 & -0.01 & $\mathrm{C}$ & $\mathrm{T}$ & 0.58 & REEP2 & $\mathrm{c}$ & $\mathrm{T}$ & n.d. \\
\hline & & & & & & & & & & & & $K D M B B$ & $\mathrm{~T}$ & $\mathrm{c}$ & n.d. \\
\hline rs10980797 & 9 & 113912553 & 0.04 & $1.75 \mathrm{E}-02$ & G & A & 0.51 & -0.02 & G & A & 0.52 & LPARI & A & G & n.d. \\
\hline rs 747249 & 11 & 130271647 & 0.04 & $2.74 \mathrm{E}-02$ & G & $A$ & 0.35 & 0.01 & G & A & 0.36 & ADAMTSS* & G & A & n.d. \\
\hline rs 10878367 & 12 & 66436097 & -0.05 & $2.04 \mathrm{E}-02$ & A & $\mathrm{T}$ & 0.73 & 0.02 & $\mathrm{~T}$ & A & 0.30 & $L L P H-A S I$ & A & $\mathrm{T}$ & n.d. \\
\hline \multirow[t]{5}{*}{ rs 12325187} & 16 & 3364997 & -0.04 & $2.27 \mathrm{E}-02$ & G & $\mathrm{c}$ & 0.69 & 0.01 & G & $\mathrm{c}$ & 0.73 & $T I G D 7$ & G & $\mathrm{c}$ & n.d. \\
\hline & & & & & & & & & & & & ZNF263 & G & c & \\
\hline & & & & & & & & & & & & LINCO00921 & $\mathrm{c}$ & G & n.d. \\
\hline & & & & & & & & & & & & ZNF200 & G & $\mathrm{c}$ & n.d. \\
\hline & & & & & & & & & & & & MTCOIP2S* & G & $\mathrm{c}$ & n.d. \\
\hline rs 4794033 & 17 & 47358481 & -0.06 & $1.68 \mathrm{E}-02$ & A & G & 0.88 & -0.02 & G & A & 0.10 & ZNF652 & A & G & n.d. \\
\hline \multirow[t]{2}{*}{ rs 1328757} & 20 & 56135199 & 0.05 & $4.73 \mathrm{E}-03$ & $\mathrm{~T}$ & $\mathrm{c}$ & 0.56 & 0.01 & $\mathrm{c}$ & $\mathrm{T}$ & 0.47 & $P C K 1$ & $\mathrm{~T}$ & $\mathrm{c}$ & \\
\hline & & & \multicolumn{5}{|c|}{ Isoprenaline simulated lipolysis } & \multicolumn{4}{|c|}{ WHRadjBMII } & \multicolumn{2}{|c|}{ eQTL (SAT) } & & Detected \\
\hline SNP & Chrom. & Pos. & Beta & $P$ & Ref & Alt & Freq & Beta & Ref & Alt & Freq & Gene & High & Low & in hASCs \\
\hline \multirow[t]{2}{*}{$\mathrm{rs} 10055995$} & 5 & 137698299 & 0.03 & $1.03 \mathrm{E}-02$ & $\mathrm{~T}$ & $\mathrm{C}$ & 0.418 & -0.01 & $\mathrm{C}$ & $\mathrm{T}$ & 0.58 & REEP2 & $\mathrm{C}$ & $\mathrm{T}$ & n.d. \\
\hline & & & & & & & & & & & & $K D M 3 B$ & $\mathrm{~T}$ & $\mathrm{c}$ & n.d. \\
\hline rs13198178 & 6 & 41702227 & -0.05 & $4.30 \mathrm{E}-02$ & $\mathrm{c}$ & G & 0.933 & 0.03 & $\mathrm{G}$ & $\mathrm{c}$ & 0.06 & TFEB & G & $\mathrm{c}$ & n.d. \\
\hline rs 10878367 & 12 & 66436097 & 0.03 & $2.64 \mathrm{E}-02$ & A & $\mathrm{T}$ & 0.727 & 0.02 & $\mathrm{~T}$ & A & 0.30 & $L L P H-A S I$ & A & $\mathrm{T}$ & n.d. \\
\hline rs 797486 & 13 & 51221618 & 0.05 & $1.04 \mathrm{E}-02$ & A & $\mathrm{c}$ & 0.153 & 0.04 & C & A & 0.89 & $E B P L$ & A & $c$ & \\
\hline rs146182298 & 14 & 52531408 & -0.09 & $2.47 \mathrm{E}-02$ & $\mathrm{~T}$ & $\mathrm{c}$ & 0.969 & 0.03 & $\mathrm{C}$ & $\mathrm{T}$ & 0.04 & NID 2* & $c$ & $\mathrm{~T}$ & \\
\hline rs11074934 & 16 & 10979440 & 0.04 & $1.15 \mathrm{E}-02$ & $\mathrm{~T}$ & $\mathrm{c}$ & 0.737 & 0.01 & $\mathrm{c}$ & $\mathrm{T}$ & 0.27 & CIITA & $\mathrm{T}$ & c & n.d. \\
\hline rs 4794033 & 17 & 47358481 & 0.05 & $2.08 \mathrm{E}-02$ & A & G & 0.883 & -0.02 & G & A & 0.10 & ZNF652 & A & G & n.d. \\
\hline \multirow[t]{5}{*}{ r59909443 } & 17 & 73308346 & -0.04 & $3.85 \mathrm{E}-03$ & $\mathrm{~T}$ & $\mathrm{c}$ & 0.780 & -0.01 & c & $\mathrm{T}$ & 0.23 & $G G . A 3$ & $\mathrm{~T}$ & c & \\
\hline & & & & & & & & & & & & MRPS7 & $\mathrm{c}$ & $\mathrm{T}$ & \\
\hline & & & & & & & & & & & & NUPS5 & $\mathrm{T}$ & C & \\
\hline & & & & & & & & & & & & $M I F 4 G D$ & $c$ & $\mathrm{~T}$ & n.d. \\
\hline & & & & & & & & & & & & GRB2 & $\mathrm{T}$ & $\mathrm{c}$ & \\
\hline rs 4646342 & 17 & 17493272 & -0.03 & $3.91 \mathrm{E}-02$ & A & G & 0.550 & -0.02 & $\mathrm{G}$ & A & 0.45 & PEMT & G & A & \\
\hline \multirow[t]{2}{*}{ rs 8126001} & 20 & 62711459 & 0.03 & $2.98 \mathrm{E}-02$ & $\mathrm{~T}$ & $\mathrm{c}$ & 0.516 & -0.02 & c & $\mathrm{T}$ & 0.49 & OPRLI & $\mathrm{T}$ & C & n.d. \\
\hline & & & & & & & & & & & & RGS19 & $\mathrm{T}$ & $\mathrm{C}$ & n.d. \\
\hline
\end{tabular}


Where Chrom. $=$ chromosome, Pos. = genome position in basepairs accoding to GRCh37, Beta refer to Alt allele, Freq $=$ frequency of Alt allele

High = allele with highest gene expression according to GTEx; n.d. = mRNA not detected in hASC undergoing in vitro differentiation to adipocytes.

* eQTL in visceral adipose tissue

\section{Figures}


452,317 SNPs associated with spontaneous lipolysis $(P<0.05)$ 463,779 SNPs associated with Isoprenaline stimulated lipolysis $(P<0.05)$

941 SNPs associated with BMI $(P<5 \times 10-8)$

346 SNPs associated with WHRadjBMI $(P<5 \times 10-8)$

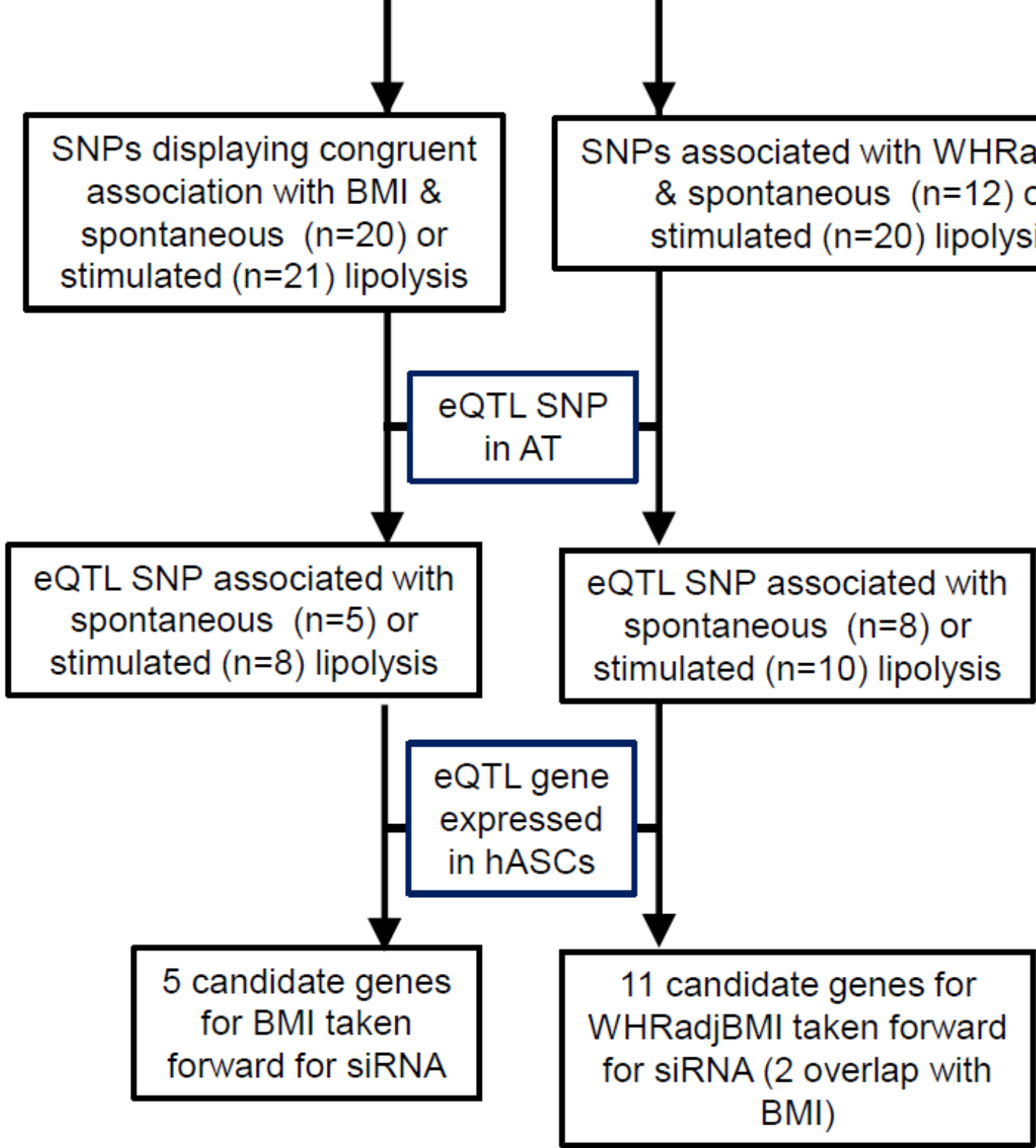

Figure 1

Flowchart describing the project. 
a

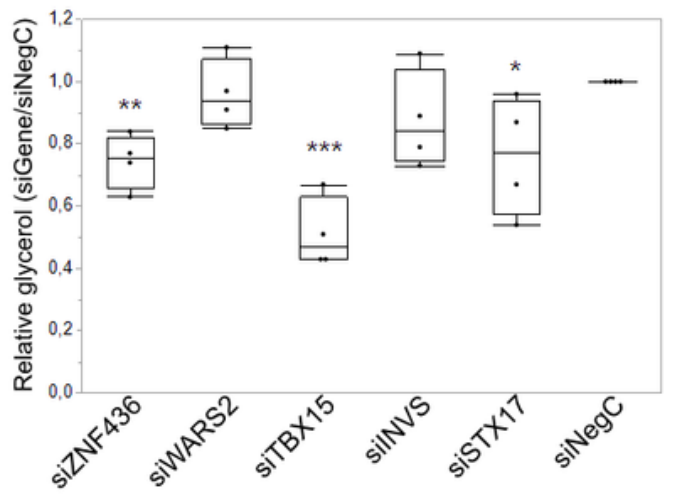

b

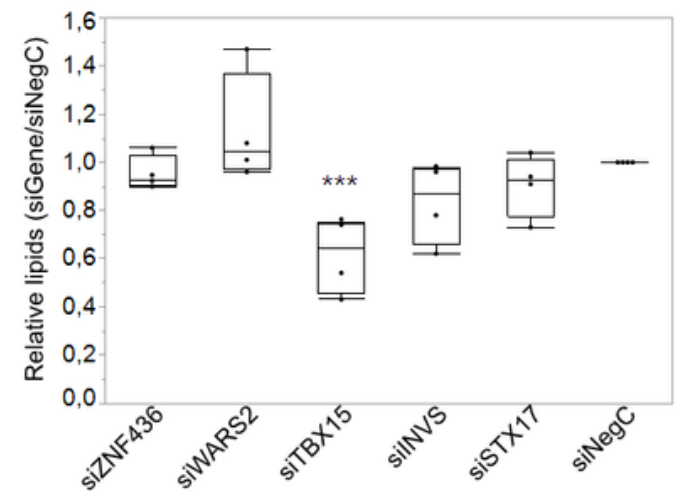

C

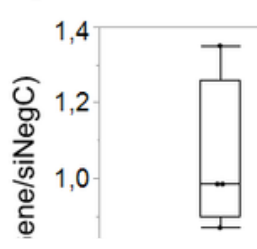

*

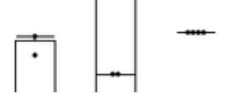

d

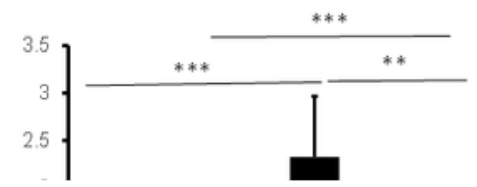

\section{Figure 2}

Effects of RNA interference-mediated knock-down on lipolysis and lipids in human adipose-derived stem cells. hASCs were transfected with control siRNA oligonucleotide (siNegC) or indicated siRNAs targeting specific genes. a-b. Expression of ZNF436, WARS2, TBX15, INVS and STX17 was knocked down using siRNA in hASCs $24 \mathrm{~h}$ prior induction of differentiation and followed until differentiation day 9 , upon which accumulated glycerol in medium was measured to assess spontaneous lipolysis (a) and accumulation of 
neutral lipids was evaluated by lipid staining (b). c, e. Expression of PCK1, EBPL, NID2, GGA3, MRPS7, NUP85, GRB2 and PEMT was knocked down using siRNA in hASCs $24 \mathrm{~h}$ prior induction of differentiation and followed until differentiation day 9 , upon which glycerol in medium was measured to assess spontaneous lipolysis (c) and accumulation of neutral lipids was evaluated in the cells (e). Results are based on four biological/independent experiments. Results were analyzed using t-test and presented in fold change \pm SD relative to negative control Neg $C$. ${ }^{\star \star *} P<0.005,{ }^{\star} * P<0.01,{ }^{*} P<0.05$. d. Expression of NUP85 was knocked down using siRNA in hASCs $24 \mathrm{~h}$ before induction of adipogenenic differentiation of hASCs. At day 9 of differentiation cells were incubated with isoprenaline (ISO) and the effects of knockdown on lipolysis were evaluated. Results are based on four biological/independent experiments. Results were analyzed using t-test and are presented as fold change \pm SD relative to $0 \mathrm{M}$ isoprenaline (ISO) of respective condition (siNegC or siNUP85). ${ }^{* \star *} \mathrm{P}<0.005$, ${ }^{*} \mathrm{P}<0.01$.

a

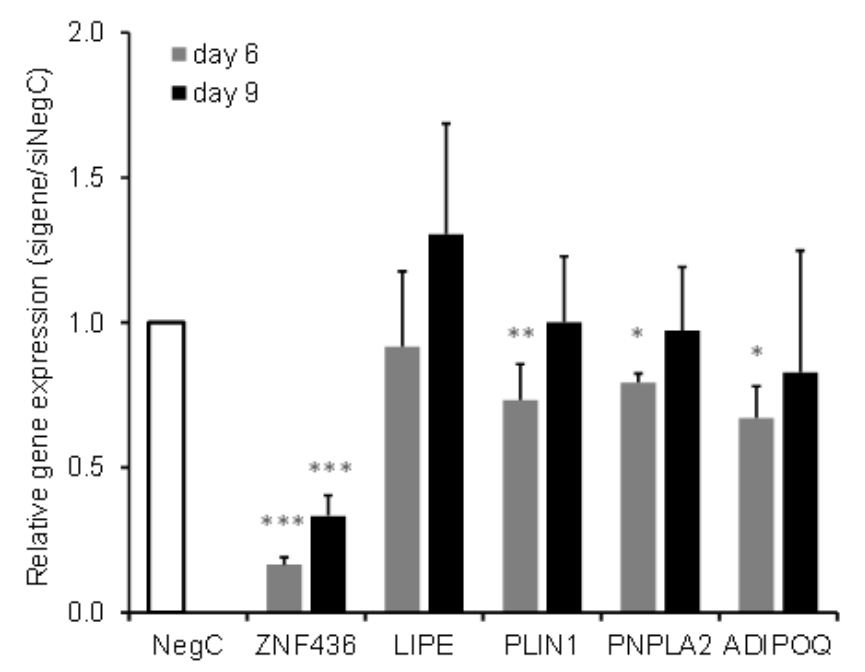

C

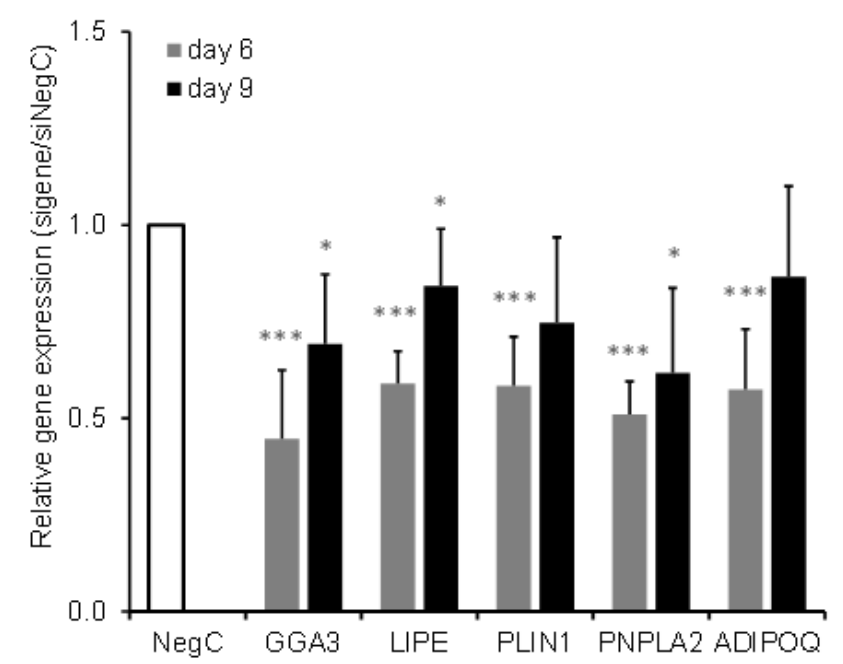

b

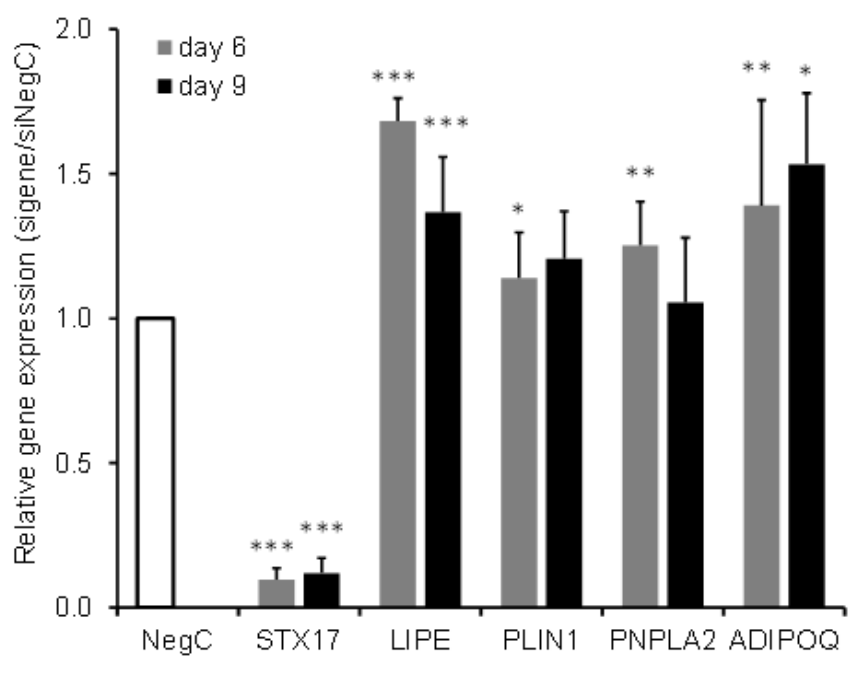

d

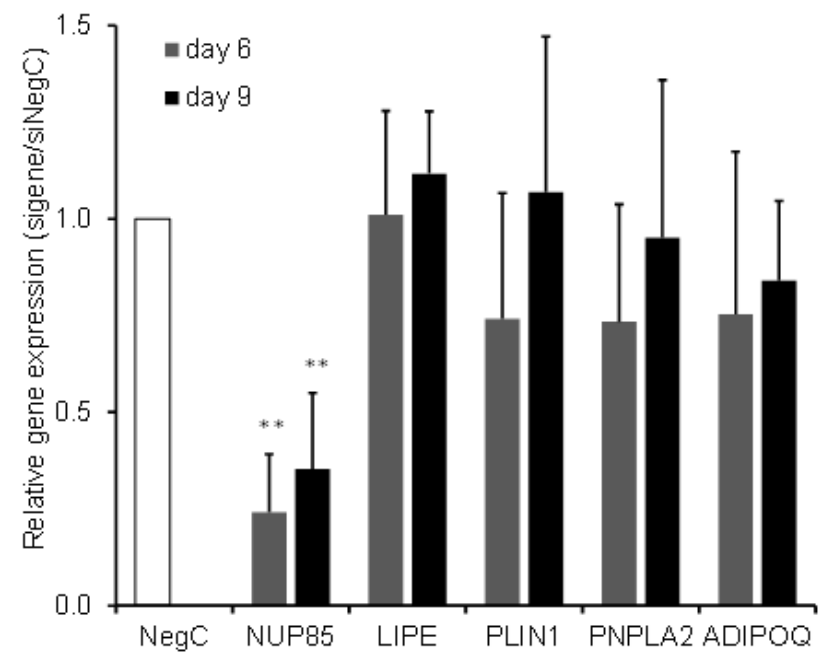

Figure 3 
Effects of RNA interference-mediated knock-down of candidate genes in human human adipose-derived stem cells. Human adipose-derived stem cells were transfected with control siRNA (siNegC) or indicated siRNAs targeting specific genes (ZNF436, STX17, GGA3 and NUP85) and followed until differentiation day 6 and 9, upon which the expression of target genes, LIPE, PLIN1, PNPLA2 and ADIPOQ was monitored. Relative gene expression was normalized to the reference gene 18s. Results were analyzed using t-test and presented as fold change \pm SD relative to control siRNA $(\mathrm{Neg} C)$ of corresponding time point $(n=6$, for GGA3 $n=11)$. ${ }^{* \star} P<0.005, * * P<0.01, * P<0.05$.

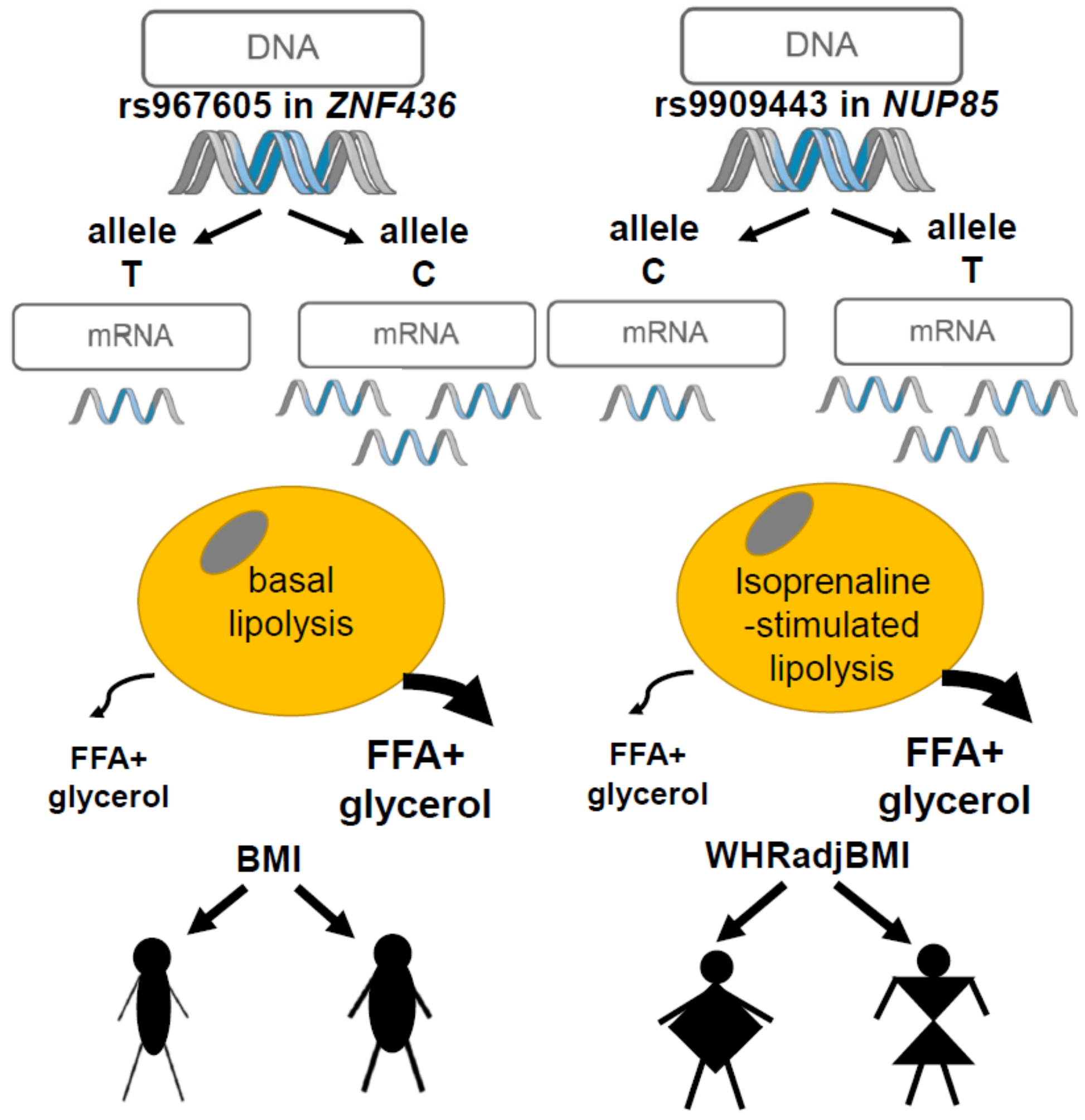


Figure 4

Summary figure demonstrating main findings for ZNF436 and NUP85.

\section{Supplementary Files}

This is a list of supplementary files associated with this preprint. Click to download.

- BMIWHRGWASSupplementalFigure1.pptx

- BMIWHRGWASSupplementalFigure2.pptx

- SupplementalFigure3.pptx

- SupplementalFigure4MR.pdf

- SupplementalFigure5MR.pdf

- BMIWHRGWASST111.pdf

- Supplementaryfigurelegends20210902.docx 